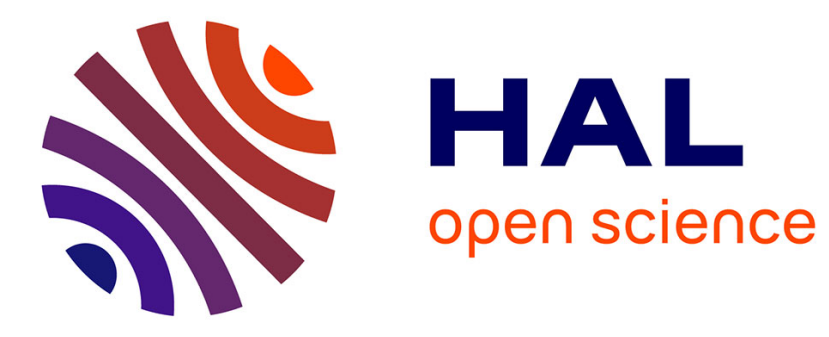

\title{
Systèmes à vide pour installations de fusion thermonucléaire
}

\author{
R. Frank, H.J. Forth
}

\section{To cite this version:}

R. Frank, H.J. Forth. Systèmes à vide pour installations de fusion thermonucléaire. Revue de Physique Appliquée, 1977, 12 (10), pp.1729-1734. 10.1051/rphysap:0197700120100172900 . jpa-00244394

\section{HAL Id: jpa-00244394 https://hal.science/jpa-00244394}

Submitted on 1 Jan 1977

HAL is a multi-disciplinary open access archive for the deposit and dissemination of scientific research documents, whether they are published or not. The documents may come from teaching and research institutions in France or abroad, or from public or private research centers.
L'archive ouverte pluridisciplinaire HAL, est destinée au dépôt et à la diffusion de documents scientifiques de niveau recherche, publiés ou non, émanant des établissements d'enseignement et de recherche français ou étrangers, des laboratoires publics ou privés. 


\title{
SYSTÈMES A VIDE POUR INSTALLATIONS DE FUSION THERMONUCLÉAIRE $(*)$
}

\author{
R. FRANK et H. J. FORTH
}

Leybold-Heraeus GmbH \& CO KG, 5000 Cologne 51, Postfach 510760, R.F.A.

\author{
(Reçu le 3 février 1977, révisé le 27 juin 1977, accepté le 8 juillet 1977)
}

\begin{abstract}
Résumé. - Les exigences relatives aux systèmes à vide pour les installations de fusion thermonucléaire sont très poussées. A l'heure actuelle, le monde entier projette ou utilise déjà des installations de fusion conçues pour la plupart d'après le principe TOKAMAK. C'est pourquoi nous nous limiterons dans cet exposé à ce modèle. L'enceinte à vide de ces installations a une géométrie toroïdale de quelques mètres de diamètre, le volume de travail étant donc de l'ordre de 10 à $200 \mathrm{~m}^{3}$, les surfaces de 10 à $10^{3} \mathrm{~m}^{2}$. Nous ne parlerons pas des exigences particulières relatives aux parois de ces enceintes mais plutôt des groupes de pompage nécessaires à leur fonctionnement. En général, le gaz à pomper est de l'hydrogène, la pression limite dans la phase de nettoyage de l'enceinte est d'environ $10^{-10}$ mbar et, dans la phase de fonctionnement, d'environ $10^{-7}$ à $10^{-9} \mathrm{mbar}$. Le groupe de pompage doit créer un vide pratiquement exempt d'hydrocarbures pour que l'enceinte ne soit pas contaminée.

Les débits requis sont de l'ordre de $10^{4}$ à $10^{5} 1 / \mathrm{s}$ pour l'enceinte d'expérimentation et de $10^{5}$ à $10^{6} 1 / \mathrm{s}$ pour les systèmes d'injection.

Le vide est créé par deux systèmes : un groupe de pompage primaire constitué de pompes primaires mécaniques avec dépresseurs Roots et de pompes turbomoléculaires qui ont pour rôle de garantir un vide primaire de quelques $10^{-5}$ à $10^{-4}$ mbar exempt d'hydrocarbures.

Le second système est un groupe de pompage à vide poussé fonctionnant dans la plupart des cas indépendamment du premier. Dans les petits postes expérimentaux, on emploie des pompes cryostatiques ou des pompes à sublimation de titane. Dans les grosses installations, on n'utilise presque exclusivement que des pompes cryostatiques dont nous parlerons en détail.

Le groupe de pompage à vide poussé doit en outre répondre aux exigences du fonctionnement par impulsions des installations de fusion thermonucléaire.

Pour les systèmes d'injection prévus dans les installations de fusion thermonucléaire, on a besoin de débits extrêmement élevés sur un petit espace de sorte que seules les pompes cryostatiques entrent en ligne de compte. Nous verrons différentes pompes qui sont actuellement en construction.
\end{abstract}

Abstract. - Thermo-nuclear plants set a special standard with respect to the vacuum system. To date all over the world fusion plants either in operation or in the design stage are built according to the TOKAMAK principle. Confirming our consideration to this type of plant the vacuum vessel is of toroidal shape of a few meters in diameter; the effective volume amounts to about 10 to $200 \mathrm{~m}^{3}$. Thus the inner surface area is 10 to $10^{3} \mathrm{~m}^{2}$. We are not dealing however in this paper with the special requirements on the properties of the surface of the inner wall, but intend to discuss the pump units that are needed for operating the TOKAMAK vacuum tank. The gas to be pumped is usually hydrogen, the required ultimate pressure in the cleaning phase of the tank is about $10^{-10} \mathrm{mbar}$, with the TOKAMAK in operation $10^{-7} \ldots 10^{-9} \mathrm{mbar}$. The absence of hydrocarbon is extremly important in order to avoid contamination. Pumping speeds required are about $10^{4} \ldots 10^{5} \mathrm{l} / \mathrm{s}$ for the tank where the experiments are carried out and $10^{5} \ldots 10^{6} \mathrm{l} / \mathrm{s}$ for the injectors.

The vacuum will be provided by two systems: a) the fore vacuum system which consists of mechanical fore-pumps and roots pumps, and of turbomolecular pumps which produce the hydrocarbon-free fore-vacuum of a few $10^{-5}$ up to $10^{-4}$ mbar. b) The high-vacuum system which in most cases operates independently of the fore-vacuum system. In small test systems turbomolecular pumps were used, but also cryopumps and titanium evaporator pumps. In larger plants almost exclusively cryopumps were used; these are discussed in detail. A special requirement concerning the high-vacuum system is its ability to cope with pulsed operation of the fusion plant.

The injectors - as used in fusion plants - require, confined to a small space, very high pumping speeds, that can be produced only by cryopumps. Our report includes the discussion of various types of such pumps presently under construction.

1. Introduction. - Les recherches sur la fusion thermonucléaire sont arrivées à un stade où on a déjà

$\left({ }^{*}\right)$ Communication présentée au Congrès National des Plasmas, 6-10 décembre 1976, Paris. beaucoup d'expériences d'importance moyenne en cours et où de grosses installations sont déjà en projet ou en construction. Les postes expérimentaux et les grosses installations de fusion nucléaire nécessitent des technologies nouvelles, ce qui concerne particulièrement le matériel sous vide [1]. Nous allons étudier 
quelques aspects de ce domaine en nous limitant toutefois aux installations fonctionnant d'après le principe TOKAMAK. Les enceintes à vide de ces installations, qui entourent le plasma, ont en général une géométrie toroïdale de quelques mètres de diamètre. Le volume de travail de ces enceintes va de 10 à $200 \mathrm{~m}^{3}$, la surface intérieure étant d'environ 10 à $10^{3} \mathrm{~m}^{2}$.

Nous n'allons pas nous occuper en détail des enceintes à vide et des problèmes qui s'y rapportent mais nous nous consacrerons surtout aux groupes de pompage nécessaires pour créer et maintenir le vide. Ces groupes de pompage doivent satisfaire à des exigences bien précises, parmi lesquelles nous citerons :

- le fait que le gaz à pomper est d'abord, au stade expérimental, l'hydrogène; par la suite, il s'agira de deutérium ou d'un mélange de deutérium et de tritium.

- Le gaz est amorcé à intervalles réguliers dans le tore. A chaque impulsion, une quantité de gaz de 1 à 600 mbar.l est admise pour une durée de 0,1 à 30 secondes. La pression d'admission se situe entre $10^{-3}$ et $10^{-5}$ mbar. Le groupe de pompage doit réduire, entre les décharges, la quantité de gaz qui évolue à des pressions entre $10^{-5}$ et $10^{-8}$ mbar.

- Une des phases opérationnelles les plus importantes est le nettoyage du système du tore avant le commencement de l'expérience. Une des opérations primordiales est le dégazage le plus complet possible des enceintes à vide, sinon la contamination du plasma par des ions étrangers serait trop forte pendant la réalisation d'expériences avec le plasma. C'est pourquoi les exigences relatives à la possibilité d'un étuvage de l'enceinte à vide et du groupe de pompage sont très poussées. Indispensables sont des joints tout métal d'une part, d'autre part des températures d'étuvage allant jusqu'à $500{ }^{\circ} \mathrm{C}$. L'objectif est d'atteindre une pression limite à la fin de la phase de nettoyage d'environ $10^{-8}$ à $10^{-10}$ mbar. En outre, on exige du groupe de pompage l'obtention d'un vide exempt de gaz étranger, en particulier d'hydirocarbure.

- Le débit-volume des pompes se calcule à partir des dimensions de l'installation expérimentale et des conditions de service envisagées. En fonction de la taille, le débit effectif pour l'hydrogène, mesuré directement sur le tore, est de $500 \mathrm{l} / \mathrm{s}$ à $15000 \mathrm{l} / \mathrm{s}$; dans les grosses installations, il peut aller jusqu'à $50000 \mathrm{l} / \mathrm{s}$ pour l'hydrogène. Sur les grosses installations, on prévoit en outre des systèmes qu'on appelle systèmes d'injection de neutres rapides et qui ont pour tâche de chauffer le plasma formé en lui fournissant l'énergie des particules injectées. Les pompes destinées à ce système d'injection doivent avoir un débit de l'ordre de 100000 à $300000 \mathrm{l} / \mathrm{s}$ pour l'hydrogène. En fonction du nombre de systèmes d'injection, le débit-volume total devra s'élever jusqu'à $6 \times 10^{6} \mathrm{l} / \mathrm{s}$ pour l'hydrogène (par exemple dans l'expérience
Joint European Torus que nous appellerons par la suite le JET).

- On doit attacher une attention particulière à la résistance au rayonnement des groupes de pompage. Le rayonnement de neutrons peut aller jusqu'à $10^{20}$ neutrons par décharge dans le cas précédent du JET.

Ces quelques conditions marginales doivent toujours être respectées lorsqu'il s'agit de concevoir des groupes de pompage primaire et à vide poussé. Nous allons maintenant passer à quelques exemples de groupes de pompage que nous avons fabriqués ou dont nous avons établi un projet.

2. Groupes de pompage primaire. - Le dispositif ASDEX est une installation de fusion de taille moyenne en cours de montage à l'Institut Max Planck de Garching. Le groupe de pompage pour le vide poussé se compose de pompes à sublimation de titane incorporées au tore.

Le groupe de pompage primaire à l'extérieur se compose de deux parties : un poste de pompage primaire avec dépresseur Roots (Fig. 1) et huit pompes

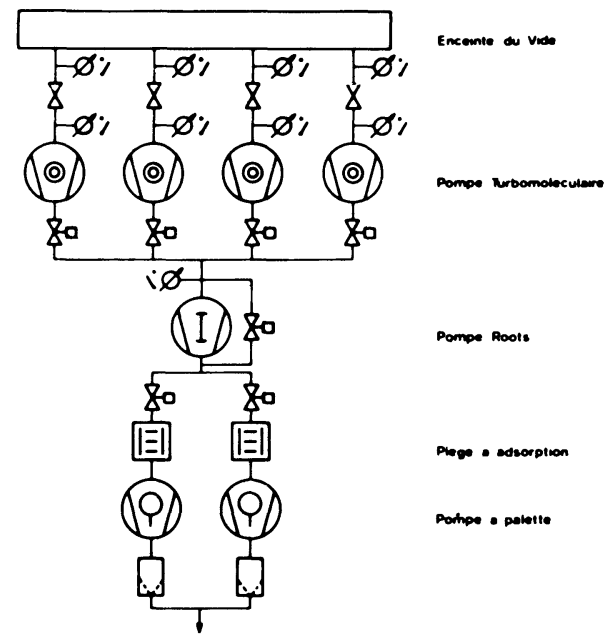

FIG. 1. - Groupe de pompage primaire pour l'expérience de fusion ASDEX.

[Fore-vacuum pump assembly for the fusion experiment ASDEX.]

turbomoléculaires TURBOVAC 3500 . Notre conception du groupe de pompage primaire serait deux pompes à piston oscillant raccordées à un dépresseur Roots par l'intermédiaire de pièges à adsorption. Le débit-volume total $S$ de cette combinaison est de $1000 \mathrm{~m}^{3} / \mathrm{h}$. Le dépresseur Roots travaille sur une canalisation de prévidage commune à laquelle sont raccordées les huit pompes turbomoléculaires. Cellesci sont reliées à la chambre du tore par l'intermédiaire de robinets-vannes à tiroir.

La figure 2 montre un assemblage semblable. Les pompes ainsi que l'unité de commande sont logées dans un châssis. 


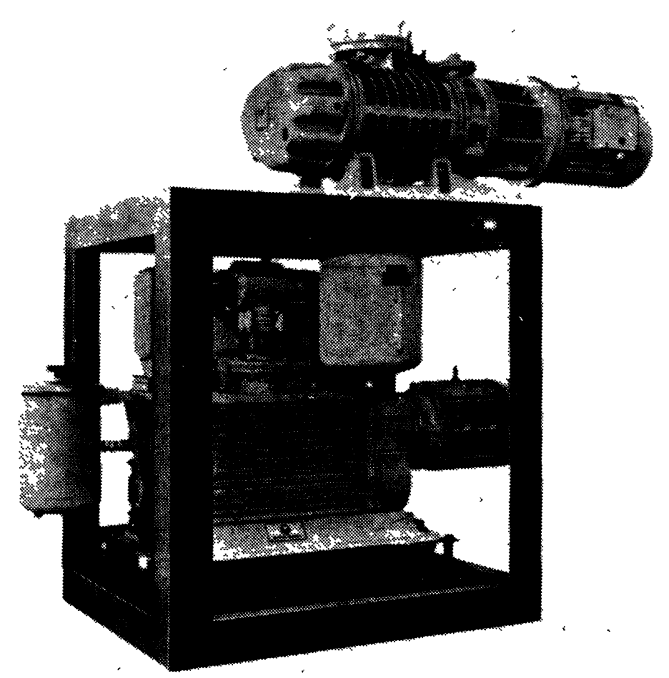

FIG. 2. - Groupe de pompage primaire; agencement conçu pour l'expérience de fusion ASDEX.

[Fore-vacuum pump assembly ; suggested for the fusion experiment ASDEX.]

Pour le prévidage des enceintes à vide, on emploie de plus en plus des pompes turbomoléculaires [2], [3]. Ce que les clients demandent, ce sont des pompes dont le débit-volume soit le plus grand possible et avec un taux de compression élevé pour l'hydrogène. Notre TURBOVAC 3500 que vous voyez sur la figure 3 remplit ces conditions. Son débit-volume $S$ est de $3500 \mathrm{l} / \mathrm{s}$, son taux de compression pour l'hydrogène supérieur à $10^{4}$. Il n'est pas besoin de rappeler que les pompes turbomoléculaires engendrent un vide pratiquement exempt d'hydrocarbure.
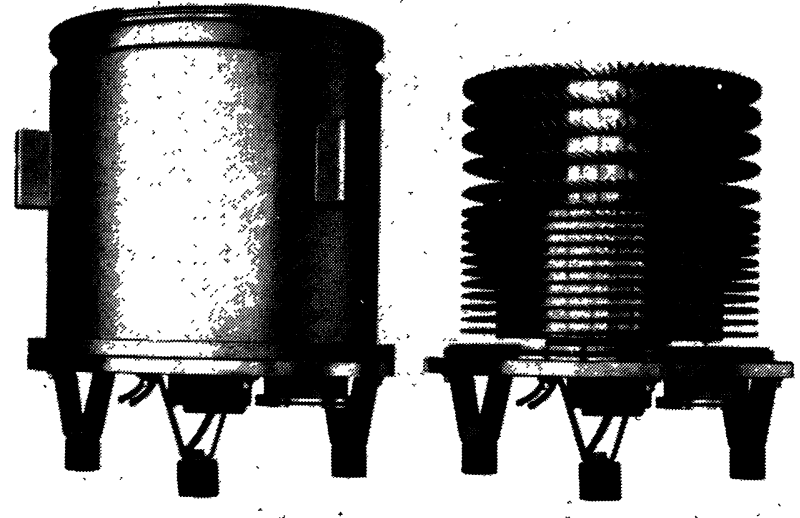

Fig. 3. - TURBOVAC 3500 , vue d'ensemble et vue sur le rotor.

[Turbomolecular pump TURBOVAC 3500 ; complete pump and view of the rotor.]
Le modèle utilisé ici est du type conventionnel à turbine avec des paliers à billes graissés à l'huile qui se trouvent dans la partie sous vide primaire de la pompe. Les expérimentateurs qui travaillent avec du tritium craignent que celui-ci ne contamine l'huile et envisagent donc d'employer un nouveau modèle de pompe turbomoléculaire à paliers magnétiques. Nous voyons sur la figure 4 une coupe de cette pompe. Le rotor est soulevé par deux paliers dynamiques radiaux et un palier dynamique axial. On n'a donc pas besoin de lubrifiant. Nous observons en outre sur cette même figure un palier sec de sécurité composé en haut de deux paliers à billes et en bas d'un palier à billes. Ceux-ci sont enduits d'un lubrifiant solide exempt d'hydrocarbure. La TURBOVAC $550 \mathrm{M}$ a un débit-volume $S$ de $530 \mathrm{l} / \mathrm{s}$ et un taux de compression pour l'hydrogène d'environ 50000 . Ce modèle convient donc particulièrement pour les installations de fusion thermonucléaire.

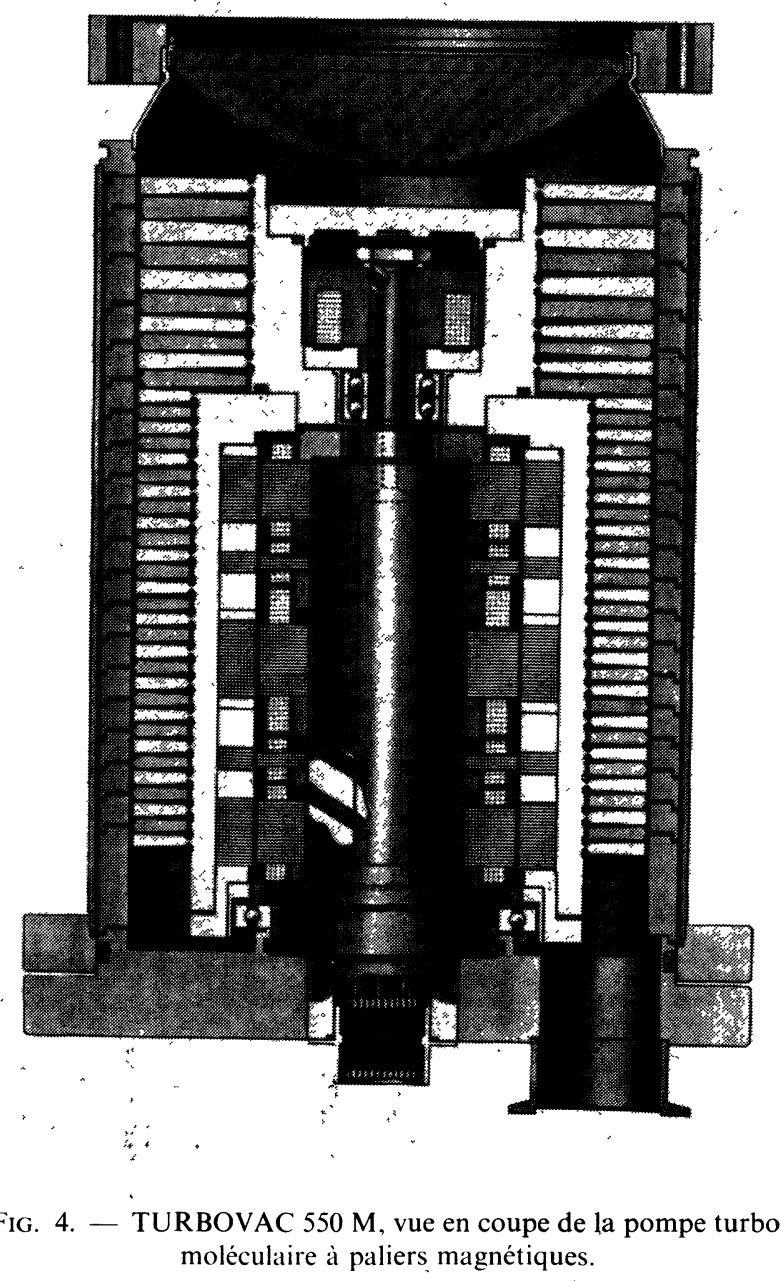

[Turbomolecular pump TURBOVAC $450 \mathrm{M}$; section through a magnetobearing turbomolecular pump.]

Pour l'expérience de fusion JET en projet, on envisage également l'utilisation d'un groupe de pompage primaire se composant de pompes turbomolé- 
culaires et de pompes primaires rotatives conventionnelles. A l'encontre du projet ASDEX, on vise ici à installer plusieurs unités indépendantes, chacune étant raccordée à une ou plusieurs pompes cryostatiques. On supprimerait alors les grands dépresseurs Roots.

3. Groupes de pompage à vide poussé. - Pour créer le vide poussé dans de grosses installations de fusion, on ne prévoit presque exclusivement que des pompes cryostatiques. Pour des postes expérimentaux moins importants, on peut également avoir recours à des pompes à sublimation de titane comme c'est le cas pour le projet ASDEX. Nous allons toutefois nous limiter ici aux pompes cryostatiques. Ces pompes offrent en effet un maximum d'avantages, entre autre la perspective d'opérer le plus économiquement. On cite des prix variant entre 1 et 2 Mark par litre/seconde de débit-volume prévu.

Nous avons déjà fait allusion au fait qu'on distingue les pompes cryostatiques destinées à la mise sous vide du tore de celles prévues pour être incorporées au système d'injection. La figure 5 montre une pompe cryostatique que nous avons proposée pour le JET [4] dans le cadre d'une étude qui nous a été confiée. Elle représente une des 16 unités qui sont reliées au tore par des raccords de pompage. Le volume du tore est d'environ $200 \mathrm{~m}^{3}$, sa surface intérieure d'environ $500 \mathrm{~m}^{2}$. Si on part d'une valeur relativement défavorable, soit d'un taux de dégazage de la surface de $10^{-12} \mathrm{mbar} .1 / \mathrm{cm}^{2} . \mathrm{s}$, le débit effectif devra être d'environ $50000 \mathrm{l} / \mathrm{s}$ pour qu'on atteigne la pression limite requise $p \approx 10^{-10}$ mbar. Du fait de la perte de charge des canalisations d'admission et du robinet-vanne à disque (voir Fig. 5), chaque pompe cryostatique devra avoir un débit pour l'hydrogène d'environ $26000 \mathrm{l} / \mathrm{s}$. Le débit des 16 unités

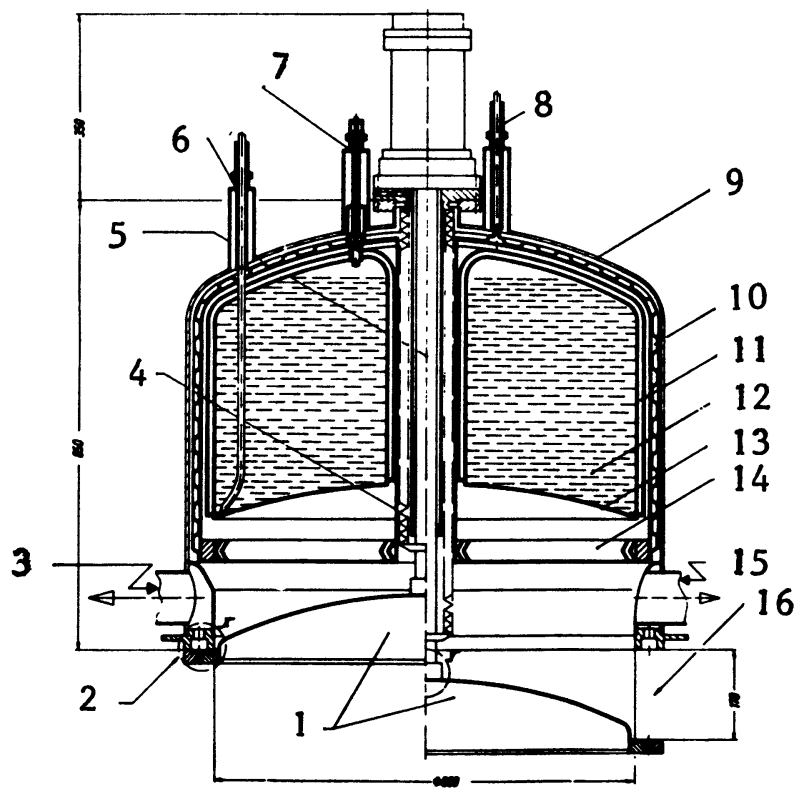

FIG. 5. - Pompe cryostatique destinée au tore du JET. [ Cryopump designed for the fusion experiment JET.] est de 420000 l/s pour l'hydrogène. Le robinet-vanne à disque monté devant la tubulure d'admission de la pompe cryostatique doit permettre d'isoler la pompe cryostatique de l'enceinte à vide pendant que le tore est en service. L'objectif est en effet de pouvoir régénérer chacune des pompes cryostatiques sans être obligé d'interrompre l'expérience. Le robinet-vanne à disque est actionné par un mécanisme à tige centrale à commande pneumatique.

Derrière le robinet-vanne à disque se trouve un baffle à chevrons refroidi à la température de l'azote liquide. Le baffle protège la surface cryogène active refroidie par de l'hélium liquide contre le rayonnement ambiant. Son coefficient de transmission pour les particules $T_{m}$ est d'environ 0,22. Le fond d'un réservoir d'hélium liquide d'une contenance de 2001 forme la surface cryogène. La surface de cette dernière est argentée pour permettre d'atteindre le coefficient d'émission le plus bas possible. La consommation d'hélium liquide est donc faible, du moins au commencement de la phase expérimentale.

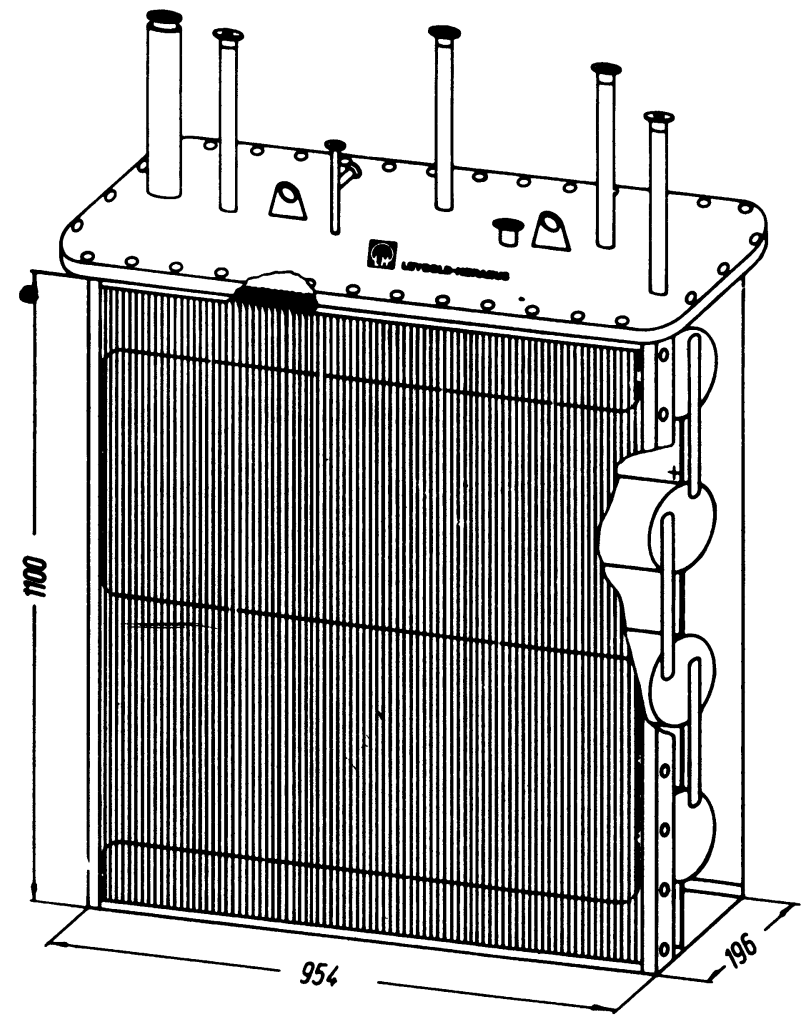

FIG. 6. - Prototype de pompe cryostatique destinée à être incorporée au système d'injection du JET.

[Prototype of a cryopump designed for operation in the JET injector system.]

La pompe cryostatique est prévue pour fonctionner pendant 7 jours, c'est-à-dire 168 heures, pendant lesquels le réservoir d'hélium n'a besoin d'être rempli qu'une fois. La température du bain d'hélium est portée à $2,5 \mathrm{~K}$ par pompage. Cette température est requise pour des opérations effectuées à $10^{-10}$ mbar.

Nous avons développé en collaboration avec le 
Laboratoire Basses Températures FHI de Berlin un prototype de pompe cryostatique que nous avons construit dans nos ateliers et qui permet d'étudier le comportement de ce genre de pompes dans les conditions particulières d'utilisation sur des installations de fusion thermonucléaire. Ce prototype se trouve au stade de la mise en route chez le FHI et nous communiquerons les résultats dès que nous les aurons.

Pour l'installation de fusion JET, on a prévu 24 systèmes d'injection; le débit-volume devra être au total de $6 \times 10^{6} \mathrm{l} / \mathrm{s}$ pour l'hydrogène. Le rapport du débit-volume des pompes cryostatiques pour les injecteurs à celui des pompes cryostatiques pour le tore est d'environ 10 à 1 , ce qui en revient à dire que ce sont les pompes destinées aux systèmes d'injection qui déterminent la puissance frigorifique à prévoir. En moyenne, la puissance frigorifique requise pour les pompes cryostatiques des systèmes d'injection est d'environ $100 \mathrm{~W}$ à $4,2 \mathrm{~K}$.

Nous sommes en train de construire un modèle de pompe cryostatique pour systèmes d'injection que nous avons nous-mêmes conçu à l'intention de l'équipe chargée de l'étude du JET. La pompe est suspendue à une bride rectangulaire par laquelle passent les conduites d'alimentation en azote liquide et en hélium liquide, ainsi que les dispositifs auxiliaires. On introduit la pompe par en haut dans une enceinte $\mathrm{du}$ système à injection. La pompe ellemême comprend un baffle à chevrons d'environ $1 \mathrm{~m}^{2}$, refroidi à $77 \mathrm{~K}$ par de l'azote liquide, et la surface cryogène placée derrière. Le débit de cet ensemble est d'environ 100000 l/s pour l'hydrogène.

La surface cryogène a une forme spéciale qui a été déterminée par l'équipe s'occupant de l'étude du JET. Ce qu'on exige, c'est une stabilité de la température dans l'espace et le temps de $\Delta t$ inférieure à $\pm 0,02 \mathrm{~K}$, qui soit garantie également dans les opérations par impulsions. En outre, il faut que la température de la surface cryogène soit réglable de 4,2 à 3,5 $\mathrm{K}$ par pompage adéquat du bain d'hélium. La durée minimale de service doit être de 8 heures. Partant de ces conditions marginales, nous avons conçu la surface cryogène de la manière suivante : derrière le baffle, nous avons placé quatre réservoirs cylindriques horizontaux, chacun d'une capacité de 9 litres d'hélium liquide. Des plaques de cuivre relient les réservoirs sur toute leur longueur, assurant ainsi un excellent contact thermique entre eux. Les parties frontales des réservoirs sont reliées entre elles par une conduite de remplissage et une canalisation commune de pompage. On peut donc régler rapidement et simultanément dans les quatre réservoirs la température de service requise et la maintenir constante même lorsque la charge thermique varie (fonctionnement par impulsions).

La figure 7 montre en coupe une autre pompe cryostatique destinée à être incorporée dans un système d'injection. Il s'agit d'une pompe cryosta-

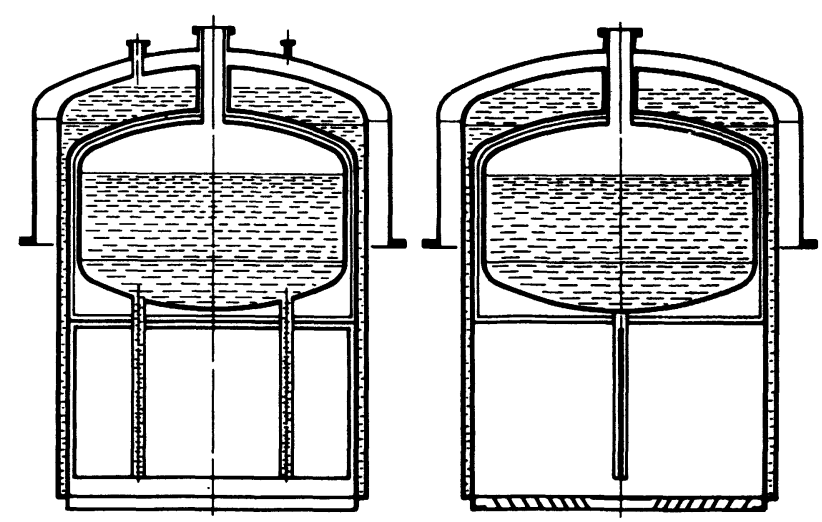

FIG. 7. - Schéma d'une pompe cryostatique destinée à un système d'injection pour DITE, d'un débit de 160000 l/s pour l'hydrogène.

[Schematic diagram of a cryopump designed for operation in an injector system for DITE. Pumping speed for $\mathrm{H}_{2}$ is $160000 \mathrm{l} / \mathrm{s}$.]

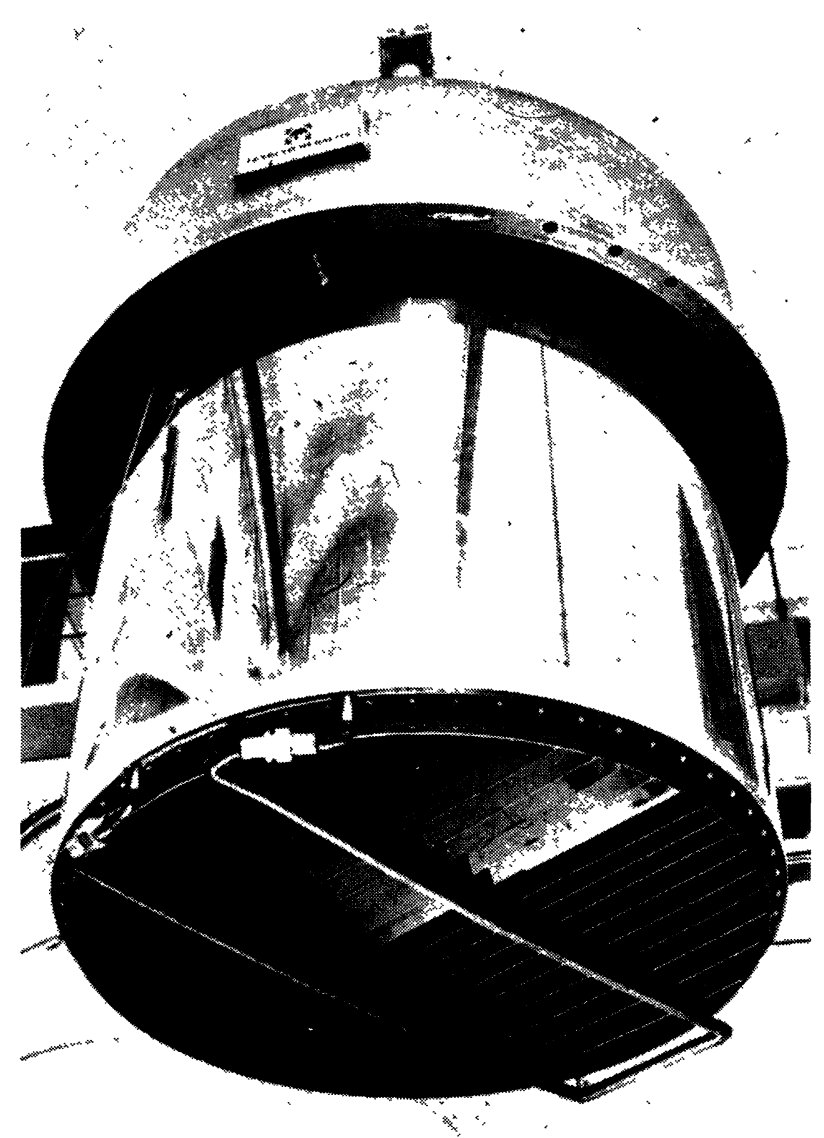

FIG. 8. - Vue d'ensemble d'une pompe destinée à un système d'injection pour DITE.

[View of the complete cryopump for the DITE injector system.]

tique en phase liquide d'un débit de $160000 \mathrm{l} / \mathrm{s}$ pour l'hydrogène que nous avons livrée aux Laboratoires Culham pour l'expérience DITE. Nous sommes en 
train de construire deux autres pompes qui seront bientôt prêtes. Les résultats feront eux-mêmes l'objet d'un exposé de sorte que nous nous contentons ici de traiter les données essentielles.

La pompe est refroidie avec de l'azote liquide et de l'hélium liquide. Le temps de garde pour le bain d'hélium liquide doit être de 7 jours, ce qui signifie que le réservoir d'hélium liquide doit avoir une capacité de plus de 300 litres. On peut dire qu'en gros la pompe est cylindrique. Elle est équipée d'un baffle à écrans parallèles ouvert de haute conductance, le but étant d'obtenir un débit très élevé avec un petit diamètre. La surface cryogène active est perpendiculaire au baffle à écrans parallèles. Elle touche le réservoir d'hélium liquide et est refroidie par conduction thermique. Les premiers résultats ont prouvé que la pompe fonctionnait parfaitement.

On voit une partie de cette pompe sur la figure 8 . On reconnaît la bride d'où sort l'écran catathermique refroidi à l'azote, à l'extrémité inférieure duquel est fixé le baffle à écrans parallèles. Ce dernier est refroidi en plus par de l'azote liquide circulant dans des canaux, ce qui assure une répartition uniforme de la température le long des écrans du baffle. On voit à la partie supérieure de la pompe différents passages pour l'alimentation en fluides cryogènes et les dispositifs auxiliaires correspondants.

4. Discussion. - Il faut attacher une importance toute particulière, lorsqu'on utilise des pompes cryostatiques dans d'assez grosses installations de fusion thermonucléaire, à l'alimentation en fluide cryogène. Tandis que la technologie pour la fabrication et le fonctionnement des pompes cryostatiques de performances élevées est déjà très avancée, on n'a pas encore développé de système d'alimentation complexe. En particulier l'alimentation des pompes en hélium liquide est discutée. Les avis sont partagés en ce qui concerne la perte de puissance cryogène (par évaporation) dans les lignes de transfert d'hélium liquide, ce qui fait que les spécialistes sont encore indécis lorsqu'il s'agit d'étudier et de concevoir des cryogénérateurs à hélium.

[1] Klipping G., Frank R., Forth H. J., Jensen K., Venus G., Proc. ICEC6, Grenoble (1976), C4.

[2] Frank R., Vak. Tech. 23 (1974) 109, Frank R., Usselmann E., BäCHLER W., Vak. Tech. 24 (1975) 78, FRANK R., J. Technol. Vide Trans. Suppl. Le Vide 157 (1972) 257.

[3] Frank R., Usselmann E., Vak. Tech. 25 (1976) 141.

[4] Frank R., Forth H. J., Lentges G., Venus G., Jensen K., KLIPPING G., Proc. ICEC6, Grenoble (1976), C1/C2. 